\title{
A RENEWAL THEOREM FOR RANDOM WALKS IN MULTIDIMENSIONAL TIME
}

\author{
J. GALAMBOS, K.-H. INDLEKOFER AND I. KÁTAI
}

\begin{abstract}
Let $X, X_{1}, X_{2}, \ldots$ be a family of integer valued, independent and identically distributed random variables with positive mean and finite (positive) variance. Let $S_{n}=X_{1}+X_{2}+\cdots+X_{n}$. The asymptotic behavior of the weighted sum $R(k)=\sum a_{n} P\left(S_{n}=k\right)$, with summation over $n \geq 1$, is investigated as $k \rightarrow+\infty$. In the special case $a_{n}=d_{r}(n)$, the number of solutions of the equation $n=n_{1} n_{2} \cdots n_{r}$ in positive integers $n_{j}, 1 \leq j \leq r, R(k)$ becomes the renewal function $Q(k)$ for a random walk in $r$-dimensional time whose terms are distributed as $X$. Under some assumptions on the magnitude of $a_{n}$ and of $A(x)=\sum_{n \leq x} a_{n}$, (i) it is shown that $R(k)$ is asymptotically distribution free as $k \rightarrow+\infty$, (ii) the proper order of magnitude of $R(k)$ is determined, and under some further restrictions on $A(x)$, (iii) a simple asymptotic formula is given for $R(k)$. From (i), the known asymptotic formula for $Q(k)$ with $r=2$ or 3 is deduced under the sole assumption of finite variance. The relaxation of previous moment assumptions requires a new inequality for the sum of the divisor function $d_{r}(n), 1 \leq n \leq x$, which by itself is of interest.
\end{abstract}

I. Introduction. Let $X, X_{1}, X_{2}, \ldots$ be a family of integer valued, independent and identically distributed random variables with positive mean $E(X)=\mu<+\infty$ and finite positive variance $V(X)=\sigma^{2}$. Let $S_{n}=X_{1}+X_{2}+\cdots+X_{n}$. Let $d_{r}(n)$ denote the number of solutions of $n=n_{1} n_{2} \cdots n_{r}$ in positive integers $n_{j}$. For a fixed integer $r \geq 2$, we consider the sum

$$
Q(k)=\sum_{n=1}^{+\infty} d_{r}(n) P\left(S_{n}=k\right) .
$$

Assume that $X$ is aperiodic in the sense that $E(\exp (i t X)) \neq 1$ for any $t \neq 0$. Then, the now classical renewal theorem (see Doob (1948), Erdös, Feller and Pollard (1949) and Chung and Pollard (1952)) states that, for $r=1$, i.e. with $d_{1}(n)=1$ for all $n, Q(k) \rightarrow 1 / \mu$ as $k \rightarrow+\infty$.

The case $r \geq 2$ has recently been the subject of active investigations. Notice that $Q(k)$ in (1.1), when $r \geq 2$, can be redefined as follows. Let $K_{r}$ be the set of all $r$-vectors $\mathbf{n}=\left(n_{1}, n_{2}, \ldots, n_{r}\right), n_{i} \geq 1$ integer, and let $X_{\mathbf{n}}, \mathbf{n} \in K_{r}$, be independent copies of $X$. Let

$$
S_{\mathbf{n}}=\sum X_{\mathbf{j}}
$$

Received by the editors March 3, 1986.

1980 Mathematics Subject Classification (1985 Revision). Primary 60K05; Secondary 60F05.

Key words and phrases. Random walk, multidimensional time, renewal theorem, weighted renewal function, inequality, asymptotic formula, divisor function. 
where the summation is over all $\mathbf{j}=\left(j_{1}, j_{2}, \ldots, j_{r}\right)$ such that $1 \leq j_{i} \leq n_{i}, 1 \leq i \leq r$. Then

$$
Q(k)=\sum_{\mathbf{n} \in K_{r}} P\left(S_{\mathbf{n}}=k\right) .
$$

Now, Ney and Wainger (1972) proved that, if $r=2$ and if $E\left(|X|^{4}\right)<+\infty$, then

$$
Q(k) \sim(\log k) / \mu \quad \text { as } k \rightarrow+\infty .
$$

M. Maejima and T. Mori (1984) replaced the moment condition by $E\left(|X|^{3}\right)<+\infty$ and proved that, for $r=2$ or 3 ,

$$
Q(k) \sim(\log k)^{r-1} /(r-1) ! \mu \quad \text { as } k \rightarrow+\infty .
$$

Their proof is based on the following form of the central limit theorem (see Petrov (1975), p. 207):

$$
P\left(S_{n}=k\right)=\phi_{n}\left(k ; \mu, \sigma^{2}\right)\left[1+\left(\beta / 6 \sigma^{2} n^{1 / 2}\right)\left(\xi_{n, k}^{3}-3 \xi_{n, k}\right)\right]+c_{n, k} / n\left(1+\left|\xi_{n, k}\right|^{3}\right),
$$

where $\beta=E\left[(X-\mu)^{3}\right]$,

$$
\phi_{n}(k)=\phi_{n}\left(k ; \mu, \sigma^{2}\right)=\exp \left(-\frac{1}{2} \xi_{n, k}^{2}\right) /(2 \pi n)^{1 / 2} \sigma,
$$

with

$$
\xi_{n, k}=(n \mu-k) / \sigma n^{1 / 2},
$$

and $c_{n, k}$ are constants such that $c_{n}=\sup _{k}\left|c_{n, k}\right| \rightarrow 0$ as $n \rightarrow+\infty$. The limitation $r=2$ and 3 is due to the lack of good asymptotic results for the sum of $d_{r}(n)$ over $n$.

In a recent paper, Galambos and Kátai (1986) gave an error term in (1.2) for $r=2$ or 3 , and proved that, for arbitrary $r \geq 2$, as $N \rightarrow+\infty$,

$$
\lim \sup \frac{1}{N} \sum_{k=N+1}^{2 N}\left|Q(k)-s\left(\log k \mu^{-1}\right)\right|<+\infty
$$

with an explicitly given polynomial $s(\cdot)$ of degree $r-1$, under the assumption that the third absolute moment of $X$ is finite. One of the main reasons for this last assumption on the third moment is the appeal to the quoted central limit theorem for first establishing that the asymptotic behavior of $Q(k)$, as $k \rightarrow+\infty$, can always be reduced to that of

$$
Q_{1}(k)=\sum_{n=1}^{+\infty} d_{r}(n) \phi_{n}(k)
$$

The relation of $Q(k)$ and $Q_{1}(k)$ is made more precise in Galambos and Kátai (1986a) under a weaker moment assumption. As a matter of fact, it is established that, whatever $r$,

$$
Q(k)=Q_{1}(k)+o(1) \quad \text { as } k \rightarrow+\infty
$$

if

$$
\int_{|x| \geq z} x^{2} d F(x)=O\left(z^{-a}\right)
$$


with a suitable $0<a<1$, where $F$ denotes the distribution function of $X$. Furthermore, Galambos and Kátai (1986a) also prove that

$$
Q_{1}(k)>c_{1}(\log k)^{r-1}
$$

with a suitable constnt $c_{1}>0$ which may depend on $r$.

The aim of the present paper is to further relax the moment assumption and to prove the following results.

THEOREM 1. Let $X$ be integer valued, aperiodic, with finite positive mean $\mu$ and finite (positive) variance $\sigma^{2}$. Then, for any fixed $r \geq 2$,

$$
Q(k)=Q_{1}(k)+o\left((\log k)^{r-1}\right) \quad \text { as } k \rightarrow+\infty .
$$

Consequently,

$$
Q(k) / Q_{1}(k) \rightarrow 1 \text { as } k \rightarrow+\infty .
$$

COROLLARY. Let $r=2$ or 3 . Then, for integer valued, aperiodic $X$ with finite positive mean $\mu$ and variance, (1.2) holds.

Note that, on account of (1.7), (1.8) indeed implies (1.9). In addition, it should be noted that (1.8) and Lemma 2 of Maejima and Mori (1984) immediately yield the corollary.

The proof of Theorem 1 is based upon the following assertions.

LEMMA 1. Under the hypothesis stated in Theorem 1

$$
\sup _{k}\left(1+\xi_{n, k}^{2}\right)\left|P\left(S_{n}=k\right)-\phi_{n}(k)\right|<\varepsilon(n) n^{-1 / 2},
$$

where $\varepsilon(n) \rightarrow 0$ as $n \rightarrow+\infty$.

For the proof see Bhattacharya and Ranga Rao (1976), Theorem 22.1, pp. 231237.

LEMMA 2. Let $\varepsilon>0$ be an arbitrary constant. Then for $x^{\varepsilon} \leq y \leq x$,

$$
\sum_{x \leq n \leq x+y} d_{r}(n)<c(r, \varepsilon) y(\log x)^{r-1},
$$

where $c(r, \varepsilon)$ is some positive constant depending on $r$ and $\varepsilon$.

We shall use Lemma 2 only with $\varepsilon=1 / 2$.

The proof of Theorem 1 is given in the next section within the framework of a more general formulation. Lemma 2, which appears to be of interest on its own, is proved in $\S 3$.

2. A renewal theorem for a class of weighted renewal functions. Let $a_{n} \geq 0, n \geq 1$, be a sequence of real numbers, and set

$$
A(x)=\sum_{n \leq x} a_{n} .
$$

Assume that

$$
a_{n}=O\left(n^{\varepsilon}\right)
$$


for every $\varepsilon>0$, where the constant implied by the order term may depend on $\varepsilon$. Furthermore, let us assume that, with some positive constants $c_{1}, c_{2}, c_{3}$ and $c_{4}$, the inequalities

$$
c_{1} h L(x) \leq A(x+h)-A(x) \leq c_{2} h L(x)
$$

and

$$
c_{3} \leq L(h) / L(x) \leq c_{4}
$$

hold with a positive function $L(x)$ for all $x \geq 1$ and $\sqrt{x} \leq h \leq x$.

Let

$$
R(k)=\sum_{n=1}^{+\infty} a_{n} P\left(S_{n}=k\right)
$$

and

$$
R_{1}(k)=\sum_{n=1}^{+\infty} a_{n} \phi_{n}(k) .
$$

We now prove the following result.

THEOREM 2. Assume that the conditions stated for $X$ in Theorem 1 and the order assumptions (2.2), (2.3) and (2.4) hold. Then

$$
R(k)=R_{1}(k)+o\left(R_{1}(k)\right) \quad \text { as } k \rightarrow+\infty .
$$

Furthermore, with suitable positive constants $c_{5}$ and $c_{6}$,

$$
c_{5} \leq R_{1}(k) / L(k) \leq c_{6}
$$

Before proceeding to the proof, let us remark that all assumptions of Theorem 2 which relate to $a_{n}$ are satisfied when $a_{n}=d_{r}(n)$ with arbitrary $r$. Indeed, $(2.2)$ is well known (see Hardy and Wright (1960) for $r=2$, which trivially extends to $r \geq 2$ by induction), and the lower inequality of $(2.3)$ with $L(x)=(\log x)^{r-1}$ is very simple to prove (see Galambos and Kátai (1986a) for details). The upper inequality of (2.3), on the other hand, is our Lemma 2. Finally, (2.4) is evident when $L(x)$ is a power of $\log x$. Consequently, Theorem 2 implies Theorem 1 . The inequalities in (2.8) are new even for the special case $a_{n}=d_{r}(n)$ for $r \geq 4$. The fact that (2.8) does not extend to an asymptotic formula without some further assumptions on the sequence $a_{n}$ is pointed out by Kawata (1961) who investigated $R(k)$ in the special case $A(x) \sim a x$ with some real number $a$.

ProOF OF THEOREM 2. We first observe that the inequality $c_{7}(\log x)^{-c}<$ $L(x)<c_{8}(\log x)^{c}$, where $c, c_{7}$ and $c_{8}$ are suitable positive constants, immediately follows frim (2.4).

Let

$$
H(n, k)=\left|P\left(S_{n}=k\right)-\phi_{n}(k)\right|
$$

and

$$
T(k)=\sum_{n=1}^{+\infty} a_{n} H(n, k)
$$


In view of the elementary inequality

$$
\left|R(k)-R_{1}(k)\right| \leq T(k),
$$

we have to show that $T(k)=o\left(R_{1}(k)\right)$, which in turn is equivalent to

$$
T(k)=o(L(k))
$$

assuming that (2.8) has been proved. We thus have to establish (2.8) and (2.11).

We first prove (2.11). From Lemma 1 we have

$$
H(n, k) \leq \varepsilon(n) / \sqrt{n}\left(1+\xi_{n, k}^{2}\right),
$$

and we may assume that $\varepsilon(n)$ tends to zero monotonically. Let $M_{1}=k / 2 \mu$ and $M_{2}=2 k / \mu$. If $n<M_{1}$, then $\xi_{n, k}^{2} \geq k^{2} / 4 \sigma^{2} n$, while for $n>M_{2}, \xi_{n, k}^{2} \gg n$. (Here, and in what follows, $a \gg b$ signifies the existence of a positive constant $c$ such that $a>c b$.) Thus, the contribution of the integers $n<M_{1}$ and $n>M_{2}$ to $T(k)$ in (2.9) can be majorized by the sums $\sum_{n<M_{1}} a_{n} \sqrt{n} / k^{2}$ and $\sum_{n>M_{2}} a_{n} / n^{3 / 2}$. On account of $(2.2)$, both sums above are $O\left(k^{-1 / 4}\right)$, and thus

$$
T(k)=\sum_{n=M_{1}}^{M_{2}} a_{n} H(n, k)+O\left(k^{-1 / 4}\right) .
$$

Because, as was pointed out earlier, $L(k)$ can go to zero at most as fast as a power of $\log k$, the error term above is $o(L(k))$. Hence, it remains to estimate the sum on the right-hand side above, and to show that

$$
T^{*}\left(k ; M_{1}, M_{2}\right)=\sum_{n=M_{1}}^{M_{2}} a_{n} H(n, k)=o(L(k)) .
$$

Let now $m_{j}=j(2 k / \mu)^{1 / 2}+M_{1}, j=0,1,2, \ldots, s$, where $s$ is determined by the inequality $m_{s} \leq M_{2}<m_{s+1}$. Then, by (2.12),

$$
T^{*}\left(k ; M_{1}, M_{2}\right) \leq \varepsilon\left(M_{1}\right) \sum_{j=0}^{s} \frac{1}{\left(1+\xi_{n_{j}, k}^{2}\right) \sqrt{m_{j}}} \sum_{m_{j} \leq n<m_{j+1}} a_{n},
$$

where $n_{j}$ is defined as an integer in $\left[m_{j}, m_{j+1}\right)$ for which $\xi_{n, k}^{2}$ takes its minimum in the interval $\left[m_{j}, m_{j+1}\right)$. To estimate the inner sum, we observe that $m_{j+1}-m_{j} \geq$ $\sqrt{m_{j}}$, and thus $(2.3)$ is applicable. This, together with (2.4), yields

$$
T^{*}\left(k ; M_{1}, M_{2}\right) \leq c_{9} \varepsilon\left(M_{1}\right) L(k) \sum_{j=0}^{s} \frac{1}{\left(1+\xi_{n_{j}, k}^{2}\right)} .
$$

The sum appearing on the right-hand side can be majorized by the convergent series $\sum 1 / j^{2}$, and thus $(2.13)$ is proved.

For establishing $(2.8)$, first note that $\phi_{n}(k) \gg n^{-1 / 2}$ in the range

$$
n \in[k / \mu-\sigma \sqrt{k}, k / \mu+\sigma \sqrt{k}] .
$$

Hence, keeping only these terms in $R_{1}(k)$, the lower inequality of (2.8) follows by an appeal to (2.3) and (2.4). For the upper inequality of (2.8) we start with showing that the contributions of those terms to $R_{1}(k)$ whose index $n$ satisfies either $n<k / \mu-\sqrt{k} \sigma(\log k)^{2}$ or $n>k / \mu+\sqrt{k} \sigma(\log k)^{2}$ are negligible. Indeed, 
for these $n$ with the additional limitation $n<2 k / \mu,\left|\xi_{n, k}\right| \gg(\log k)^{2}$ as well as $\left|\xi_{n, k}\right| \gg(\log n)^{2}$, while for $n \geq 2 k / \mu,\left|\xi_{n, k}\right| \gg \sqrt{n}$. Hence, in view of $(2.2)$, the total contribution of these terms to $R_{1}(k)$ is smaller than an arbitrary negative power of $k$, which is negligible in terms of $L(k)$ (recall that it can go to zero at most as fast as a negative power of $\log k$ ). Next, we split up the remaining values of $n$ into intervals of the type $[k / \mu-\sigma(j+1) \sqrt{k}, k / \mu-\sigma j \sqrt{k})$ and $[k / \mu+\sigma j \sqrt{k}, k / \mu+\sigma(j+1) \sqrt{k})$ where $0 \leq j \leq(\log k)^{2}$. Over the $j$ th interval of either type above, $c j^{2} \leq \xi_{n, k}^{2} \leq c^{*}(j+1)^{2}$ with some positive constants $c$ and $c^{*}$. Thus, for these $n$,

$$
\Phi_{n}(k) \ll k^{-1 / 2} \exp \left(-c^{*}(j+1)^{2}\right)
$$

and their contribution to $R_{1}(k)$ is of the magnitude

$$
k^{-1 / 2} \exp \left(-c^{*}(j+1)^{2}\right)[A(k / \mu+(j+1) \sigma \sqrt{k})-A(k / \mu+j \sigma \sqrt{k})]
$$

where $A(x)$ is the function defined in (2.1). Now, upon utilizing (2.3) and (2.4), the summation over $j$ of these contributions to $R_{1}(k)$ yields the upper inequality of (2.8). This completes the proof of Theorem 2 .

For the validity of $(2.7)$, condition (2.3) can be weakened at the expense of assuming more than just the finiteness of the second moment of $X$. In fact, with no change in the proof of the corresponding result in Galambos and Kátai (1986a), the following result can be obtained.

THEOREM 3. Let $a_{n} \geq 0$ satisfy (2.2). Let $L(x)$ be a positive function for which (2.4) holds. Assume that $A(x)<c x L(x)$ with some positive constant $c$ and that the lower inequality of (2.3) is valid. Let $X$ satisfy the conditions of Theorem 2 as well as the condition $\int_{|x| \geq z} x^{2} d F(x)=O\left(z^{-a}\right)$ with a suitable constant $0<a<1$, where $F(x)$ is the distribution function of $X$. Then (2.7) holds. (2.3)

In order to give an asymptotic formula for $R_{1}(k)$ we have to assume more than

THEOREM 4. Assume that conditions (2.2), (2.3) and (2.4) hold for $a_{n} \geq 0$. Furthermore assume that there exists a positive function $\rho(x)$, tending to zero monotonically, such that

$$
(A(x+h)-A(x)) / h L^{*}(x) \rightarrow 1 \quad(\text { as } x \rightarrow+\infty)
$$

uniformly in $h \in(\rho(x) \sqrt{x}, \sqrt{x})$, where $L^{*}(x)$ is a very slowly varying function in the sense that, as $x \rightarrow+\infty$,

$$
L^{*}(Y(x)) / L^{*}(x) \rightarrow 1 \quad \text { whenever }(\log Y(x)) /(\log x) \rightarrow 1 .
$$

Then, as $k \rightarrow+\infty$,

$$
R_{1}(k) \sim(1 / \mu) L^{*}(k / \mu) .
$$

ProOF. From (2.3) and (2.14) it follows that $L(x)$ and $L^{*}(x)$ have the same order of magnitude, i.e. $L(x) \ll L^{*}(x)$ and $L^{*}(x) \ll L(x)$. Now, let $u(k)$ be a function tending to infinity with $k$ and satisfying $u(k)=O(\log k)$. Let

$$
J=\left[N_{1}, N_{2}\right], \quad N_{1}=k / \mu-\sigma \sqrt{k} u(k), \quad N_{2}=k / \mu+\sigma \sqrt{k} u(k) .
$$

By elementary estimates, upon utilizing (2.3), we get

$$
\sum_{n \notin J} a_{n} \phi_{n}(k)=o\left(L^{*}(k)\right) \quad \text { as } k \rightarrow+\infty .
$$


Thus, as $k \rightarrow+\infty$,

$$
R_{1}(k)=R_{1}^{*}(k)+o\left(L^{*}(k)\right)
$$

where

$$
R_{1}^{*}(k)=\sum_{n \in J} a_{n} \phi_{n}(k)
$$

Choose an integer $M$ such that $\rho(n) \sqrt{n} \leq M \leq \sqrt{n}$ for each $n \in J$. It is easy to see that

$$
\phi_{n}(k)=\frac{1}{M} \sum_{j=0}^{M-1} \phi_{n+j}(k)+o\left(\phi_{n}(k)\right) \quad(k \rightarrow+\infty)
$$

uniformly in $n \in J$. Hence,

$$
R_{1}^{*}(k)=\frac{1}{M} \sum_{n \in J} a_{n} \sum_{j=0}^{M-1} \phi_{n+j}(k)+o\left(R_{1}^{*}(k)\right),
$$

which, by changing the order of summation, becomes

$$
R_{1}^{*}(k)=\sum_{m=N_{1}}^{N_{2}+M} \phi_{m}(k) \frac{1}{M}\left[\sum_{t=0}^{M-1} a_{m-t}\right]^{*}+o\left(R_{1}^{*}(k)\right),
$$

where the star at the end of the inner sum means that the summation extends only to those $m$ for which $m-t \in J$. This implies restriction only if either $m<N_{1}+M$ or $m>N_{2}$. The total contribution of these terms, however, does not exceed

$$
L^{*}(k) \sum_{N_{1} \leq m \leq N_{1}+M} \phi_{m}(k)+L^{*}(k) \sum_{N_{2} \leq m \leq N_{2}+M} \phi_{m}(k),
$$

which is $o\left(L^{*}(k)\right)$. Therefore, since, in view of (2.14),

$$
\frac{1}{M} \sum_{t=0}^{M-1} a_{m-t}=(1+o(1)) L^{*}\left(\frac{k}{\mu}\right)
$$

uniformly in $m \in J$,

$$
R_{1}^{*}(k)=\left\{\sum_{m=N_{1}+M}^{N_{2}} \phi_{n}(k)\right\} L^{*}\left(\frac{k}{\mu}\right)+o\left(R_{1}^{*}(k)\right) .
$$

This now completes the proof upon observing that the sum appearing on the right side converges to $1 / \mu$ as $k \rightarrow+\infty$ (see formula (34) in Galambos and Kátai (1986) and the hint for its proof).

Asymptotic formulas have been developed only in a few special cases (see W. L. Smith (1958), Kawata (1961) and Greenwood et al. (1982)) for $R(k)$. Several other papers deal with general renewal functions $R(k)$ whose assumptions on $a_{n}$, however, are unrelated to the current investigation (our assumption (2.2) is essential in relation to renewal problems in multidimensional time). In fact, the example given in Remark (b) on p. 568 by Embrechts et al. (1984) clearly shows this claim: it is demonstrated there that, under our assumption (2.2), their conclusion, and earlier related results, cannot be applied. 
3. Proof of Lemma 2. By the nature of Lemma 2, its proof is of a quite different character when compared with the rest of the paper. The basic ideas we shall use here can be found in the paper of Linnik and Vinogradov (1957).

Clearly, it is sufficient to prove the assertion for $y=x^{\varepsilon}$. Let the integers $b_{n}, n=$ $1,2, \ldots,[y]$, be defined by the formula $b_{n}=[x]+n$, where $[z]$ signifies the integer part of $z$. For an integer $m$, let $p(m)$ denote the smallest and $P(m)$ the largest prime factor of $m$. For every $b_{n}$, let $b_{n}=c_{n} r_{n}$, where $P\left(c_{n}\right) \leq y^{1 / 2}$ and $p\left(r_{n}\right)>y^{1 / 2}$. Since $d_{r}(m)$ is multiplicative, i.e., $d_{r}\left(m_{1} m_{2}\right)=d_{r}\left(m_{1}\right) d_{r}\left(m_{2}\right)$ for coprime $m_{1}$ and $m_{2}$, and, for primes $p$ and integers $a \geq 1$,

$$
d_{r}\left(p^{a}\right)=\left(\begin{array}{c}
a+r-1 \\
r-1
\end{array}\right)
$$

we have that $d_{r}\left(b_{n}\right) \ll d_{r}\left(c_{n}\right)$. Hence, for Lemma 2, we have to develop an estimate of the sum

$$
B=\sum_{n \leq y} d_{r}\left(c_{n}\right)
$$

Now, upon noting that

$$
d_{r}\left(c_{n}\right)=\sum_{m_{1}} d_{m_{2}=c_{n}} d_{r-1}\left(m_{1}\right)
$$

we can write

$$
B=B_{1}+B_{2}
$$

where

$$
B_{i}=\sum_{n \leq y} \sum_{i} d_{r-1}\left(m_{1}\right), \quad i=1,2
$$

with $\sum_{i}$ signifying summation over $m_{1}$ satisfying $m_{1} m_{2}=c_{n}$ and $m_{1} \leq y$ in $\sum_{1}$ while $m_{1}>y$ in $\sum_{2}$. The sum $B_{1}$ can easily be estimated. By interchanging summations, we have

$$
\begin{aligned}
B_{1} & \leq \sum_{m_{1} \leq y} d_{r-1}\left(m_{1}\right) \sum_{\substack{n \leq y \\
c_{m} \equiv 0\left(\bmod m_{1}\right)}} 1 \\
& \ll y \sum_{m_{1} \leq y} \frac{d_{r-1}\left(m_{1}\right)}{m_{1}} \ll y(\log y)^{r-1} .
\end{aligned}
$$

The estimation of $B_{2}$ is much more involved, and we do it in several steps. Let $m_{1}=p_{1} p_{2} \cdots p_{s}$ be the prime decomposition of $m_{1}$ with $p_{1} \leq p_{2} \leq \cdots \leq p_{s}$. Let $t$ be defined so that $t=p_{1} p_{2} \cdots p_{k} \leq y$ and $t p_{k+1}>y$. Hence, $t$ is well determined by $m_{1}$. Let us now split $B_{2}$ into two parts, $B_{2}=B_{2,1}+B_{2,2}$, say, according as $P(t) \leq \log x$ or $P(t)>\log x$.

Since, for fixed $n$ and $t$,

$$
\sum_{\substack{t\left|m_{1} \\ m_{1}\right| c_{n}}} d_{r-1}\left(m_{1}\right) \ll x^{\eta}
$$


with arbitrary $\eta>0, B_{2,1} \ll x^{\eta} y \sum 1 / t$, where the summation is extended over those $t$ for which $y^{1 / 2} \leq t \leq y$ and $P(t) \leq \log x$. For estimating this sum we appeal to the following well known result of Rankin

$$
\sum_{\substack{t \leq y \\ P(t) \leq \log x}} 1 \ll 2^{(2 \log x) / \log \log x} .
$$

We thus have

$$
B_{2,1}=O(y) .
$$

Let now $P(t)>\log x$. It is obvious that $d_{r-1}(u v) \leq d_{r-1}(u) d_{r-1}(v)$ for all integers $u$ and $v$. Thus, for fixed $n$ and $t$,

$$
\sum_{\substack{m_{1}\left|c_{n} \\ t\right| m_{1}}} d_{r-1}\left(m_{1}\right) \leq d_{r-1}(t) \prod_{q>p_{k}}\left(1+d_{r-1}(q)\right),
$$

where $q$ runs over the prime divisors of $c_{n} / t$. Since $c_{n} \leq x+y<2 x$, we get

$$
\sum_{m_{1} \mid c_{n}} d_{r-1}\left(m_{1}\right) \leq d_{r-1}(t) r^{(\log x) /\left(\log p_{k}\right)} .
$$

Hence,

$$
\begin{aligned}
B_{2,2} & \leq \sum_{1} d_{r-1}(t) r^{(\log x) /(\log P(t))} \sum_{\substack{c_{n} \\
\equiv 0 \\
n \leq y}} 1 \\
& \ll y \sum_{1} \frac{d_{r-1}(t)}{t} r^{(\log x) /(\log P(t))}=y B_{3},
\end{aligned}
$$

where $\sum_{1}$ denotes summation over $t$ with $y^{1 / 2} \leq t \leq y$ and $P(t)>\log x$.

We split $B_{3}=B_{3,1}+B_{3,2}$, where in $B_{3,1}, P(t)<x^{\gamma}$ with some $\gamma>0$, which may depend on $r$ and $\varepsilon$, and in $B_{3,2}, P(t)>x^{\gamma}$.

Note that, in $B_{3,2},(\log x) /(\log P(t))$ is bounded. Thus, if we write $P(t)=p$, $t=p t^{*}$, then

$$
B_{3,2} \ll \sum_{x^{\gamma<p<y}} \frac{1}{p} \sum^{*} \frac{d_{r-1}\left(t^{*}\right)}{t^{*}}
$$

where $\sum^{*}$ is summation over $t^{*}$ such that $P\left(t^{*}\right) \leq p$ and $y^{1 / 2} p^{-1}<t^{*}<y p^{-1}$. The sum involving $t^{*}$ is easily seen to be $O\left((\log x)^{r-1}\right)$. The well-known asymptotic formula

$$
\sum_{p \leq z} \frac{1}{p}=\log \log z+C+o(1) \quad(z \rightarrow+\infty)
$$

thus yields

$$
B_{3,2}=O\left((\log x)^{r-1}\right) .
$$

We finally estimate $B_{3,1}$. Writing again $t=p t^{*}$, where $p=P(t)$,

$$
B_{3,1} \ll \sum_{\log x<p \leq x^{\gamma}} \frac{1}{p} r^{(\log x) / \log p} \sum^{* *} \frac{d_{r-1}\left(t^{*}\right)}{t^{*}}
$$


where $\sum^{* *}$ denotes summation over $t^{*}$ such that $y^{1 / 2} \leq t \leq y$ and $p \leq x^{\gamma}$. We further increase above if we sum over all $t^{*}$ with $y^{1 / 4} \leq t^{*}$, namely, the mentioned limitations in $t$ and $p$ imply this bound for $t^{*}$ if $\gamma$ is chosen sufficiently small. We, of course, keep the additional restriction $P\left(t^{*}\right) \leq p$. Since $t^{*} / y^{1 / 4} \geq 1$, we further increase if we plug in the additional factor $\left(t^{*} / y^{1 / 4}\right)^{\delta}$ with a suitably chosen $\delta>0$. This last step again is an idea of Rankin [10]. Now, if we choose

$$
\delta=\frac{1}{3}(\log p)^{-1} \log \frac{\log y^{1 / 4}}{\log p}
$$

it follows (see Indlekofer (1980))

$$
\begin{aligned}
\sum^{* *} \frac{d_{r-1}\left(t^{*}\right)}{t^{*}} & \ll \exp \left\{\sum_{\substack{q \leq p \\
q \text { prime }}} \frac{r-1}{q}-c \frac{\log y^{1 / 4}}{\log p} \log \frac{\log y^{1 / 4}}{\log p}\right\} \\
& \leq \exp \left[(r-1) \log \log p-c \frac{\log x^{\varepsilon / 4}}{\log p} \log \frac{\log x^{\varepsilon / 4}}{\log x^{\gamma}}\right]
\end{aligned}
$$

with a positive constant $c$. Thus

$$
B_{3,1} \ll \sum_{\log x<p \leq x^{\gamma}} \frac{1}{p} \exp \left[(r-1) \log \log p+\frac{\log x}{\log p} u_{r}(\varepsilon, \gamma)\right],
$$

where $u_{r}(\varepsilon, \gamma)=\log r-(c \varepsilon / 4) \log (\varepsilon / 4 \gamma)$. Upon choosing $\gamma$ small enough, such that $u_{r}(\varepsilon, \gamma) \leq-1$, summation over $p$ in the estimate (3.9) of $B_{3,1}$ yields

$$
B_{3,1} \ll \exp \left[(-1 / \gamma)\left(\log x^{\gamma}\right)^{r-1}\right],
$$

where the trivial estimate $-(\log x) /(\log p) \leq-1 / \gamma$ as well as the well-known estimate

$$
\sum_{p \leq x^{\gamma}} \frac{(\log p)^{r-1}}{p} \ll\left(\log x^{\gamma}\right)^{r-1} \quad(r \geq 2)
$$

were utilized. Hence, since

$$
\begin{aligned}
B & =B_{1}+B_{2}=B_{1}+B_{2,1}+B_{2,2} \leq B_{1}+B_{2,1}+y B_{3} \\
& \leq B_{1}+B_{2,1}+y\left(B_{3,1}+B_{3,2}\right),
\end{aligned}
$$

the estimates $(3,4),(3.6),(3.10)$ and (3.8) establish Lemma 2.

\section{REFERENCES}

1. R. N. Bhattacharya and R. Ranga Rao, Normal approximation and asymptotic expansions, Wiley, New York, 1976.

2. K. L. Chung and H. Pollard, An extension of renewal theory, Proc. Amer. Math. Soc. 3 (1952), 303-309.

3. J. L. Doob, Renewal theory from the point of view of the theory of probability, Trans. Amer. Math. Soc. 63 (1948), 422-438.

4. P. Embrechts, M. Maejima and E. Omey, A renewal theorem of Blackwell type, Ann. Probab. 12 (1984), 561-570.

5. P. Erdös, W. Feller and H. Pollard, A property of power series with positive coefficients, Bull. Amer. Math. Soc. 55 (1949), 201-204.

6. J. Galambos and I. Kátai, A note on random walks in multidimensional time, Math. Proc. Cambridge Philos. Soc. 99 (1986), 163-170. 
7. __ Some remarks on random walks in multidimensional time, Proc. 5th Pannonian Sympos. on Math. Statist. (Visegrád, Hungary, 1985), J. Mogyoródi et al., eds., Reidel, Dordrecht, 1986a.

8. P. Greenwood, E. Omey and J. L. Teugels, Harmonic renewal measures, Z. Wahrsch. Verw. Gebiete 59 (1982), 391-409.

9. G. H. Hardy and E. M. Wright, An introduction to the theory of numbers, Oxford, 1960.

10. K.-H. Indlekofer, A mean-value theorem for multiplicative functions, Math. Z. 172 (1980), 255-271.

11. T. Kawata, A theorem of renewal type, Kodai Math. Sem. Rep. 13 (1961), 185-194.

12. Yu. V. Linnik and A. I. Vinogradov, An estimate of the sum of the number of divisors in some intervals of an arithmetical progression, Uspehi Mat. Nauk 12 (76) (1957), 277-280.

13. M. Maejima and T. Mori, Some renewal theorems for random walks in multidimensional time, Math. Proc. Cambridge Philos. Soc. 95 (1984), 149-154.

14. P. Ney and S. Wainger, The renewal theorem for a random walk in two-dimensional time, Studia Math. 46 (1972), 71-85.

15. V. V. Petrov, Sums of independent random variables, Springer-Verlag, Heidelberg, 1975.

16. W. L. Smith, Renewal theory and its ramifications, J. Roy Statist. Soc. (B) 20 (1958), 243-302.

Department of Mathematics, TU 038-16, Temple University, Philadelphia, PENNSYLVANIA 19122

FACHBEREICH MATH.-INFORMATIK, UNiversität-GeSAMThoChSCHUle, WARBURGer Strasse 100, D-4790 PADERBorN, WeSt Germany

Institute of Mathematics, L. EÖtvös University, Budapest, MuzeUm Korut 6-8, H-1088, HUNGARY 